\title{
Biosynthesis of an Anti-Addiction Agent from the lboga Plant
}

\author{
Scott C. Farrow, ${ }^{\dagger}$ Mohamed O. Kamileen, ${ }^{\dagger}$ Lorenzo Caputi, ${ }^{\dagger}$ Kate Bussey, ${ }^{\ddagger}$ Julia E. A. Mundy,
} Rory C. McAtee, ${ }^{\S}$ Corey R. J. Stephenson, ${ }^{\S}{ }^{\circledR}$ and Sarah E. O’Connor*, ${ }^{* \dagger}$

${ }^{\dagger}$ Department of Natural Product Biosynthesis, Max Planck Institute of Chemical Ecology, Hans-Knöll-Straße 8, 07745 Jena, Germany

${ }^{\ddagger}$ Department of Biological Chemistry, John Innes Centre, Norwich Research Park, Norwich NR4 7UH, United Kingdom

${ }^{\S}$ Willard Henry Dow Laboratory, Department of Chemistry, University of Michigan, 930 North University Avenue, Ann Arbor, Michigan 48109, United States

\section{Supporting Information}

ABSTRACT: (-)-Ibogaine and (-)-voacangine are plant derived psychoactives that show promise as treatments for opioid addiction. However, these compounds are produced by hard to source plants, making these chemicals difficult for broad-scale use. Here we report the complete biosynthesis of $(-)$-voacangine, and de-esterified voacangine, which is converted to (-)-ibogaine by heating, enabling biocatalytic production of these compounds. Notably, (-)-ibogaine and (-)-voacangine are of the opposite enantiomeric configuration compared to the other major alkaloids found in this natural product class. Therefore, this discovery provides insight into enantioselective enzymatic formal Diels-Alder reactions.

$\mathrm{T}$ reatment of opiate addiction remains challenging, with over 45,000 people in the United States dying in 2017 and a $500 \%$ increase in yearly opioid overdose deaths since the year 2000. ${ }^{1}$ (-)-Ibogaine (1) (Figure 1A), a plant-derived iboga-type alkaloid, has anti-addictive properties that were discovered by Howard Lotsof in 1962 when he noticed that ingesting this compound mitigated heroin cravings and acute opiate withdrawal symptomatology. ${ }^{2,3}$ Although the toxicity of (-)-ibogaine (1) has slowed its formal approval for addiction treatment in many countries, increased knowledge of its mode of action, side-effects, and the discovery of (-)-ibogaine (1) analogs clearly indicates its potential as an anti-addictive agent. $^{2-4}$ The plant that synthesizes (-)-ibogaine (1), Tabernanthe iboga (Iboga), is difficult to cultivate, prompting interest in developing biocatalytic methods for (-)-ibogaine (1) production.

While the biosynthesis of the (+)-iboga-type alkaloid scaffold was recently elucidated, ${ }^{5}$ biosynthesis of the antipodal (-)-ibogaine (1) remained unknown. Here we show that $(-)$-ibogaine (1) biosynthesis uses the same starting substrate as observed in (+)-iboga biosynthesis, but the key formal Diels-Alder cyclization step proceeds via a distinct mechanism to generate the reduced iboga alkaloid $(-)$-coronaridine (2) (Figure 1A). We further demonstrate that enzymatically generated (-)-coronaridine (2) can be 10-hydroxylated and 10-O-methylated ${ }^{6}$ to form (-)-voacangine (3), and treatment of (-)-voacangine (3) with a T. iboga esterase reported here, followed by heating, yields (-)-ibogaine (1). This biocatalytic production strategy may facilitate sustainable and enhanced production of (-)-ibogaine (1) along with less toxic analogs and, moreover, reveals how two closely related enzyme systems generate two optical series via a formal Diels-Alder reaction.

A transcriptome of $T$. iboga was previously obtained using seeds gifted by the Ibogaine Alliance. ${ }^{6}$ Upon discovery of the pathway for the structurally related, antipodal iboga alkaloid (+)-catharanthine (4) from the plant Catharanthus roseus (Figure 1A), ${ }^{5}$ we hypothesized that T. iboga homologues of these $C$. roseus enzymes were responsible for biosynthesis of the (-)-iboga scaffold. (+)-Catharanthine (4) is synthesized by oxidation of stemmadenine acetate $(6)$ by precondylocarpine acetate synthase (PAS) to yield precondylocarpine acetate (7), which is reduced by dihydroprecondylocarpine acetate synthase (DPAS) to give dihydroprecondylocarpine acetate (8). Dihydroprecondylocarpine acetate (8) undergoes elimination of acetic acid to yield the iminium form of dehydrosecodine (9a), which then undergoes isomerization to its enamime form (9b), followed by a formal Diels-Alder cyclization catalyzed by catharanthine synthase (CS), an $\alpha / \beta$ hydrolase homologue, to yield (+)-catharanthine (4) (Figure $1 \mathrm{~B}$, red box). We identified, cloned, and heterologously expressed three homologues of PAS (flavin dependent oxidases), two of DPAS (medium chain alcohol dehydrogenases), and two of CS ( $\alpha / \beta$ hydrolase homologues) from the T. iboga transcriptome.

The three PAS enzymes (T. iboga precondylocarpine acetate synthases 1-3, TiPAS1, TiPAS2, TiPAS3, respectively) each had the same biochemical function as the homologue involved in (+)-catharanthine (4) biosynthesis, CrPAS, namely oxidation of stemmadenine acetate (6) to precondylocarpine acetate (7) (Figure 1B, Figure S1). ${ }^{5}$ All subsequent experiments were performed with CrPAS, for which an optimized expression system had been developed. ${ }^{5}$ Next, T. iboga dihydroprecondylocarpine acetate synthase 1 (TiDPAS1) (76.3\% sequence identity to CrDPAS involved in catharanthine biosynthesis) and T. iboga dihydroprecondylocarpine acetate synthase 2 (TiDPAS2) ( $86.3 \%$ sequence identity to CrDPAS) were tested with CrPAS and stemmadenine acetate (6). The expected reduced product, dihydroprecondylocarpine acetate (8) (Figure 1B), is unstable and is not directly

Received: June 7, 2019

Published: July 31, 2019 


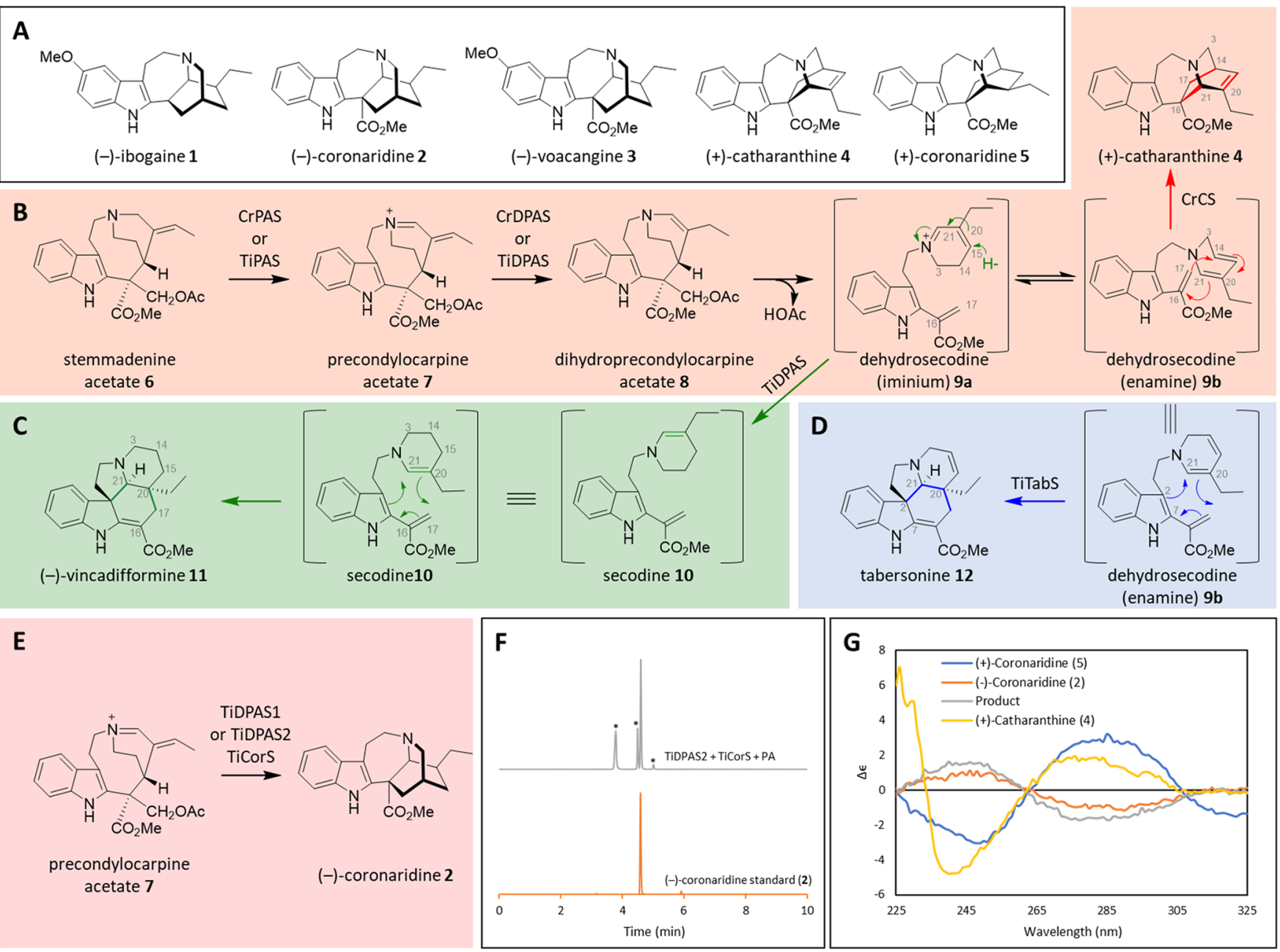

Figure 1. (+) and (-)-Iboga alkaloids. (A) Anti-addiction agents (-)-ibogaine (1) and (-)-voacangine (3) are antipodal to (+)-catharanthine (4), a precursor to the anticancer drug vincristine. (B) Biosynthesis of $(+)$-catharanthine (4). It is assumed that dehydrosecodine iminium 9a is initially formed, and it then tautomerizes to the enamine form $9 \mathbf{b}$. (C) Biosynthesis of (-)-vincadifformine (11). (D) Biosynthesis of (-)-tabersonine (12). (E) Biosynthesis of the reduced iboga alkaloid (-)-coronaridine (2) directly from precondylocarpine acetate (7). (F) LC-MS chromatogram showing formation of (-)-coronaridine (2) after incubation of precondylocarpine acetate (7) $(50 \mu \mathrm{M})$ with TiDPAS2 (1 $\mu \mathrm{M})$, TiCorS $(5 \mu \mathrm{M})$, and NADPH (8 equiv). Peaks marked with an asterisk are uncharacterized side products $(\mathrm{m} / z$ 339) that decomposed during isolation attempts. (G) CD spectra of enzymatically produced coronaridine compared to authentic standards.

observed. In the presence of limiting amounts of the nicotinamide adenine dinucleotide phosphate (NADPH) cofactor required by DPAS (1 substrate equivalent), a compound with $m / z 337$ was instead detected (Figure S2). This product, which had been previously observed with CrDPAS, ${ }^{5}$ is likely an isomer of dehydrosecodine which forms after desacetoxylation of dihydroprecondylocarpine acetate (8) (Figure 1B). Surprisingly, in the presence of excess NADPH cofactor ( $>8$ substrate equivalents), addition of either TiDPAS1 or TiDPAS2 generated the alkaloid vincadifformine (11) (Figures S3-6), which is formed through Diels-Alder cyclization of reduced dehydrosecodine (secodine (10)) (Figure 1C, green box). We hypothesize that with excess NADPH, TiDPAS1/2 over-reduces precondylocarpine acetate (7) to form secodine (10), which, in the absence of a dedicated cyclase enzyme, cyclizes spontaneously via a DielsAlder mechanism to form vincadifformine (11). Biomimetic syntheses have shown that a $( \pm)$-vincadifformine analog readily forms from secodine (10), potentially due to the propensity of secodine (10) to adopt the conformation required for this Diels-Alder cyclization. ${ }^{7,8}$ The vincadiffor- mine (11) observed in the CrPAS/TiDPAS reactions could be the result of a non-enzymatic cyclization, though surprisingly, $\mathrm{CD}$ spectra indicate that this reaction product is enantiomerically enriched (-)-vincadifformine (11) (Figure S7). Thus, binding of secodine (10) to TiDPAS1/2 may provide an enantiomerically enriched cyclization product. ${ }^{9}$

Finally, we tested the function of the two observed T. iboga CS homologues, which could be responsible for the key cyclization step to the (-)-iboga enantiomer. Addition of one of these homologues to assays containing stemmadenine acetate (6), CrPAS, and TiDPAS1/TiDPAS2 and varying amounts of NADPH, yielded the alkaloid tabersonine (12), which forms through an alternative Diels-Alder cyclization mode of dehydrosecodine $(9)^{5}$ (Figure 1D, blue box, Figure S8), and this enzyme was thus named T. iboga tabersonine synthase (TiTabS, $72.5 \%$ sequence identity to previously identified tabersonine synthase from C. roseus, CrTS). T. iboga contains tabersonine $(\mathbf{1 2})$ and numerous tabersonine derived alkaloids, ${ }^{10}$ so identification of this enzyme activity, which has previously been observed in C. roseus, was expected. Incubation of stemmadenine acetate (6) with CrPAS, TiDPAS1/ 


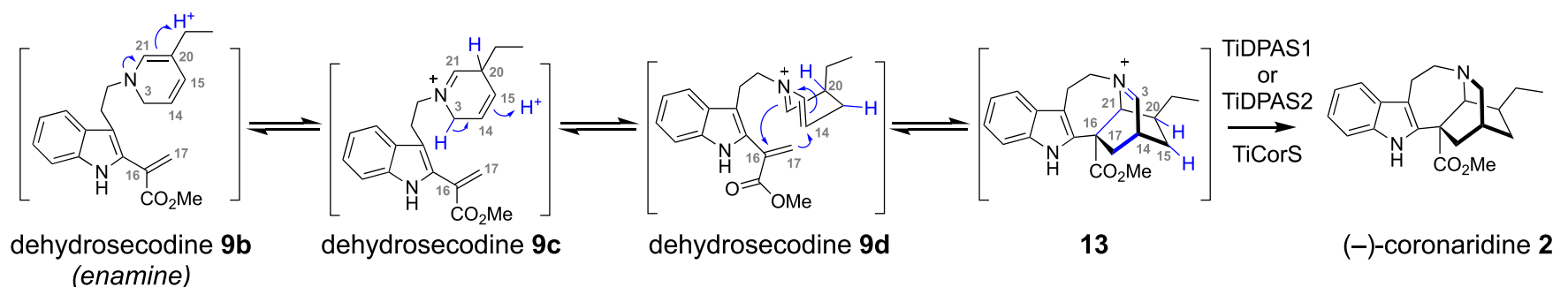

Figure 2. Biosynthesis of (-)-coronaridine (2) by TiDPAS1/2 and TiCorS. One plausible chemical mechanism for formation of the $(-)$-coronaridine $(2)$ scaffold that is consistent with experimental evidence.

TiDPAS2 or precondylocarpine acetate (7) with TiDPAS1/ TiDPAS2, excess NADPH, and the second CS homologue from $T$. iboga led to the formation of the reduced iboga alkaloid coronaridine (2) (Figure 1E), as evidenced by mass fragmentation and comparison to an authentic standard (Figure 1F, Figures S9-13). Although several side products were also observed in this in vitro enzymatic reaction (Figure $1 \mathrm{~F}$ ), coronaridine (2) was the major product. This T. iboga CS homologue was thus named TiCorS (coronaridine synthase, $71.9 \%$ sequence identity to $\mathrm{CrCS}$ ). To assign the stereochemistry of coronaridine (2), the enzymatic product was isolated and subjected to $\mathrm{CD}$ analysis, which upon comparison to previous literature reports as well as authentic standards of (-)-coronaridine (2) (isolated from Tabernaemontana divaricata $)^{6}$ and $(+)$-coronaridine (5) (obtained from total synthesis $)^{11}$ (Figure 1G) indicated that the enzymatic product is $(-)$-coronaridine $(2)$. Therefore, the biosynthetic pathways for both $(+)$ and (-)-iboga alkaloid scaffolds have now been elucidated.

We hypothesized how the (-)-coronaridine (2) enantiomer might form in this system. (-)-Catharanthine could be formed from dehydrosecodine (9), analogous to (+)-catharanthine (4) biosynthesis in $C$. roseus (e.g., Figure 1B), and then subsequently reduced to form (-)-coronaridine (2). However, $(-)$-catharanthine (4) is not observed as an intermediate in these enzymatic assays nor has ( - -) catharanthine (4) been identified from natural sources. Alternatively, while it is obvious how vincadifformine (11) can be formed directly from secodine (10) (Figure 1C), there is no logical mechanism by which any secodine (10) isomer can be cyclized to form (-)-coronaridine (2). Therefore, we hypothesize that secodine (10) also does not act as an intermediate in (-)-coronaridine (2) biosynthesis. To gather evidence for an alternative mechanism, we isolated the dehydrosecodine-like product of precondylocarpine acetate (7) and TiDPAS $1 / 2$ that is formed under limiting NADPH conditions $(9, m / z$ 337), which was also observed with DPAS from $C$. roseus. ${ }^{5}$ This compound 9 can be incubated with TiTabS to generate tabersonine (12), thus confirming it to be the dehydrosecodine substrate for the cyclases (Figure S14). We incubated 9 with TiCorS, and, instead of observing the coronaridine product directly, we observed an intermediate product, $13(\mathrm{~m} / z$ 337). This product was isolated in partially purified form and was then added to TiDPAS $1 / 2$ and NADPH ( 8 equiv), resulting in the formation of (-)-coronaridine (2) (Figure S15). Although 13 was too unstable to be structurally characterized, this experiment nevertheless suggests a reaction order and mechanism in which TiDPAS1/2 initially reduces precondylocarpine acetate (7) to generate $9 b$, which undergoes tautomerization (9cd) ${ }^{12,13}$ followed by a formal $[4+2]$ cyclization $^{14}$ to yield iminium 13. Iminium 13 would then be reduced by TiDPAS1/
2 to form (-)-coronaridine (2) (Figure 2). In further support of this mechanism, when assays with 9 , TiCorS, TiDPAS2, and $\mathrm{NADPH}$ ( 8 equiv) were performed in $\mathrm{D}_{2} \mathrm{O}$, a compound coeluting with $(-)$-coronaridine (2) with two additional atomic mass units was observed (Figure S16). Notably, when CS and TS were assayed in $\mathrm{D}_{2} \mathrm{O}$, no isotopic label was incorporated (Figure S16). This provides further support that the mechanism of TiCorS, which requires tautomerization of the dihyropyridine ring, is distinct from the mechanism of CS and TS, which proceeds directly from $9 \mathbf{b}$. This highlights that isomerization of dehydrosecodine provides the capacity for generating further structural diversity beyond (+)-catharanthine (4) and (-)-tabersonine (12). In short, this combination of cyclase, tautomerization, and reductase activity generates an additional scaffold beyond the previously reported $(+)$-catharanthine (4) and (-)-tabersonine (12).

We next tested whether enzymatically synthesized (-)-coronaridine (2) could be used to generate $(-)$-voacangine (3) and (-)-ibogaine (1). We incubated the TiCorS/TiDPAS product with the previously identified ibogaine enzymes $\mathrm{I} 10 \mathrm{H}$ and N10OMT, which respectively 10-hydroxylate and 10-Omethylate (-)-coronaridine (2) to generate (-)-voacangine (3). ${ }^{6}$ Consistent with our structural assignment of (-)-coronaridine (2), incubation of the TiCorS/TiDPAS enzymatic product with these downstream enzymes yielded a product that was identical to an authentic standard of $(-)$-voacangine (3) (Figure 3, Figure S17). Not surprisingly, incubation of $\mathrm{I} 10 \mathrm{H}$ and N10OMT with synthetic (+)-coronaridine $(5)$ gave only trace amounts of product (Figure S18), highlighting the selectivity of the downstream enzymes to the stereochemistry of the substrate. In a well-established semisynthetic process, $(-)$-voacangine (3) is subjected to a basic saponification to remove the methyl ester and then heated to facilitate decarboxylation. ${ }^{15}$ The T. iboga transcriptome revealed three homologues of polyneuridine aldehyde esterase (PNAE), an enzyme involved in the de-esterification and decarboxylation of ajmalan-type alkaloids found in other Apocynaceae plants, ${ }^{16}$ which had a similar co-expression profile as TiPAS1, TiDPAS2, and TiCorS. Incubation of T. iboga polyneudridine aldehyde esterase-like 1 (TiPNAE1) with $(-)$-voacangine (3) yielded a product with a mass consistent with the de-esterified compound 15, which then slowly converted to (-)-ibogaine (1) (Figure 3). Heating of the de-esterified (-)-voacangine (15) product increased the efficiency and speed of this conversion (Figure S19), suggesting that this decarboxylation is non-enzymatic, and a dedicated decarboxylase may be involved in planta. Regardless, enzymatic (-)-voacangine (3) provides an effective semisynthetic starting material for (-)-ibogaine (1). Although TiPNAE1 could also de-esterify (-)-coronaridine (2), decarboxylation did not occur even after heating, suggesting that the electron-donating methoxy group 


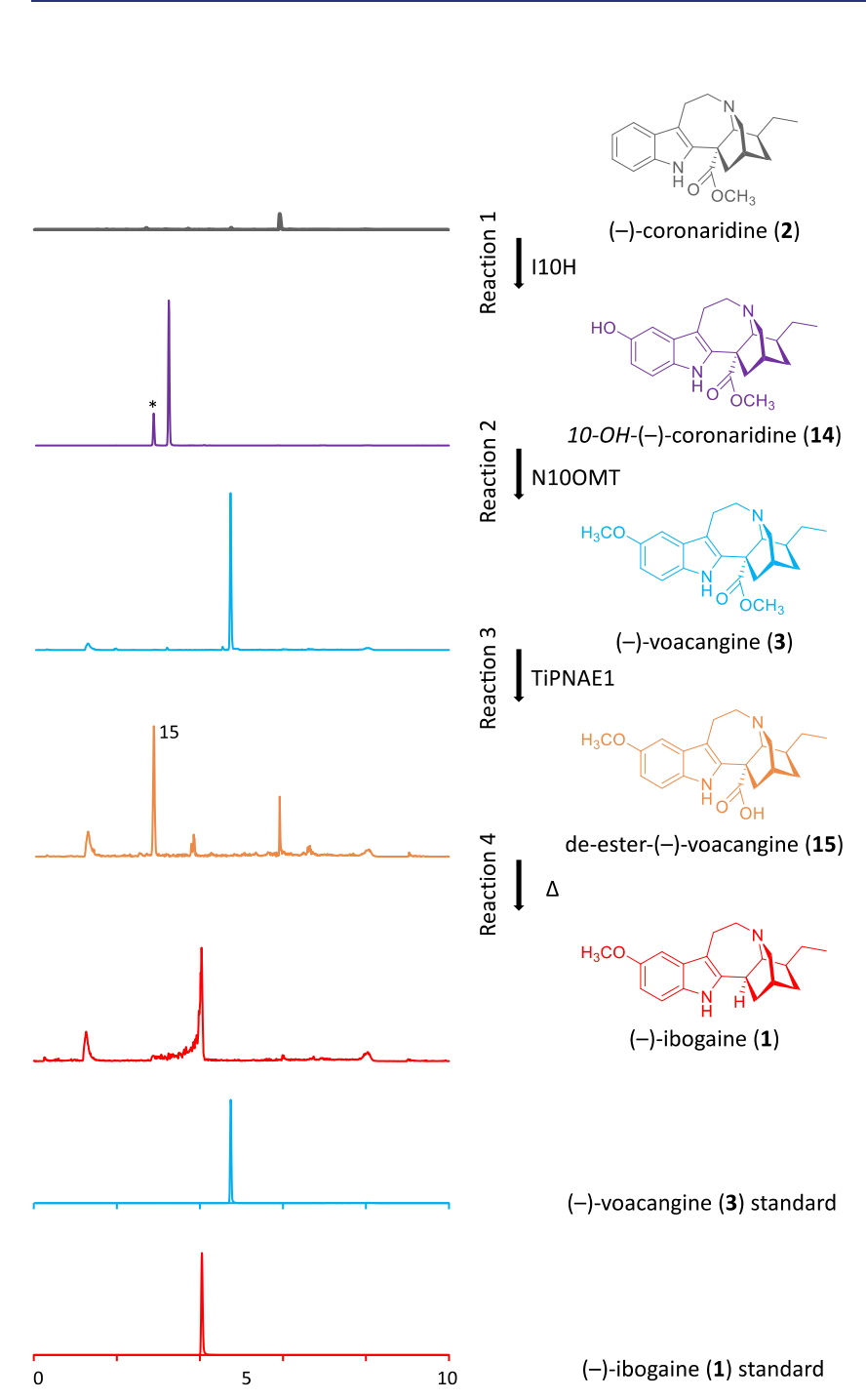

Figure 3. Formation of (-)-ibogaine (1) from enzymatically generated (-)-coronaridine (2). Peaks marked with an asterisk were products of endogenous yeast enzymes present in cultures expressing $\mathrm{I} 10 \mathrm{H}$.

on the indole of (-)-voacangine (3) is required for spontaneous decarboxylation (Figure S20).

(-)-Ibogaine (1) has a storied history dating back hundreds of years to the Congo Basin and the Bwiti religion, though it was the serendipitous discovery of the anti-addictive properties of (-)-ibogaine $(\mathbf{1})$ that captured the attention of modern medicine. Here we report the biosynthesis of $(-)$-voacangine (3) and an esterase that may improve semisynthesis of (-)-ibogaine (1). In addition to its extraordinary bioactivity, (-)-ibogaine (1) provides an opportunity to compare the formal Diels-Alder enzymatic synthesis of $(-)$ and (+)-iboga enantiomers. The discovery and initial investigation of TiCorS suggest a possible mechanism for this cyclization and a basis for future enzyme engineering for this emerging class of cyclases. There are approximately 100 iboga alkaloids identified in nature, ${ }^{10}$ both of + and - optical series, and discovery of this pathway now provides full access to both medicinally important scaffolds, ${ }^{17}$ in addition to providing the first biocatalytic method for production of the anti-addictive alkaloid (-)-ibogaine (1).

\section{ASSOCIATED CONTENT}

\section{(5) Supporting Information}

The Supporting Information is available free of charge on the ACS Publications website at DOI: 10.1021/jacs.9b05999.

Data S1: Protein identifications (XLSX)

Materials and methods, Figures S1-S20, Tables S1-S3, refs $1-6$ (PDF)

\section{AUTHOR INFORMATION}

\section{Corresponding Author}

*oconnor@ice.mpg.de

ORCID

Corey R. J. Stephenson: 0000-0002-2443-5514

Sarah E. O’Connor: 0000-0003-0356-6213

\section{Funding}

We acknowledge ERC 788301 (S.E.O.), EMBO ALTF 8462016 (S.C.F), and NSF Graduate Fellowship (DGE 1256260) (R.C.M.).

\section{Notes}

The authors declare no competing financial interest.

\section{ACKNOWLEDGMENTS}

We gratefully acknowledge Dr. Kenneth Alper for the introduction to this compound and to Jonathan Dickerson of the Ibogaine Alliance for seeds. We thank Jakob Franke, Benjamin Lichman, and Matthew DeMars for intellectual contributions, Gerhard Saalbach and Carlo de-Oliveira-Martins for protein mass spectrometry and David Lawson and Clare Stevenson for helpful discussions. We thank Andrew Davis (John Innes Centre) for the photograph of Iboga in the graphical abstract.

\section{REFERENCES}

(1) Scholl, L.; Seth, P.; Kariisa, M.; Wilson, N.; Baldwin, G. Drug and Opioid-Involved Overdose Deaths - United States, 2013-2017. MMWR. Morb. Mortal. Wkly. Rep. 2018, 67 (5152), 1419-1427.

(2) Alper, K. R. Ibogaine: A Review. Alkaloids. Chem. Biol. 2001, 56, $1-38$.

(3) Alper, K. R.; Lotsof, H. S.; Kaplan, C. D. The Ibogaine Medical Subculture. J. Ethnopharmacol. 2008, 115 (1), 9-24.

(4) Alper, K.; Bai, R.; Liu, N.; Fowler, S. J.; Huang, X.-P.; Priori, S. G.; Ruan, Y. HERG Blockade by Iboga Alkaloids. Cardiovasc. Toxicol. 2016, 16 (1), 14-22.

(5) Caputi, L.; Franke, J.; Farrow, S. C.; Chung, K.; Payne, R. M. E.; Nguyen, T.-D.; Dang, T.-T. T.; Soares Teto Carqueijeiro, I.; Koudounas, K.; Dugé de Bernonville, T.; Ameyaw, B.; Jones, D. M.; Vieira, I. J. C.; Courdavault, V.; O’Connor, S. E. Missing Enzymes in the Biosynthesis of the Anticancer Drug Vinblastine in Madagascar Periwinkle. Science 2018, 360 (6394), 1235-1239.

(6) Farrow, S. C.; Kamileen, M. O.; Meades, J.; Ameyaw, B.; Xiao, Y.; O'Connor, S. E. Cytochrome P450 and O-Methyltransferase Catalyze the Final Steps in the Biosynthesis of the Anti-Addictive Alkaloid Ibogaine from Tabernanthe Iboga. J. Biol. Chem. 2018, 293 (36), 13821-13833.

(7) Mizoguchi, H.; Oikawa, H.; Oguri, H. Biogenetically Inspired Synthesis and Skeletal Diversification of Indole Alkaloids. Nat. Chem. 2014, 6 (1), 57-64.

(8) Danieli, B.; Lesma, G.; Palmisano, G.; Passarella, D.; Silvani, A. Aspidosperma Alkaloids Cyclization of Secodine Intermediate: Synthesis of ( \pm )-3-Oxovincadifformine Ethyl Ester. Tetrahedron 1994, 50 (23), 6941-6954.

(9) Qu, Y.; Safonova, O.; De Luca, V. Completion of the Canonical Pathway for Assembly of Anticancer Drugs Vincristine/Vinblastine in Catharanthus Roseus. Plant J. 2019, 97 (2), 257-266. 
(10) Richardson, P. M.; Herz, W.; Grisebach, H.; Kirby, G. W. Progress in the Chemistry of Organic Natural Products. Volume 42. Brittonia 1983, 35 (2), 187.

(11) Beatty, J. W.; Stephenson, C. R. J. Synthesis of (-)-Pseudotabersonine, (-)-Pseudovincadifformine, and (+)-Coronaridine Enabled by Photoredox Catalysis in Flow. J. Am. Chem. Soc. 2014, 136 (29), 10270-10273.

(12) Böhm, S.; Kuthan, J. Ab Initio MO-SCF Study of Prototropic Transformations of Non-Substituted Dihydropyridines. Collect. Czech. Chem. Commun. 1982, 47 (10), 2735-2745.

(13) Stout, D. M.; Meyers, A. I. Recent Advances in the Chemistry of Dihydropyridines. Chem. Rev. 1982, 82 (2), 223-243.

(14) Buonora, P.; Olsen, J.-C.; Oh, T. Recent Developments in Imino Diels-Alder Reactions. Tetrahedron 2001, 57 (29), 60996138.

(15) Jenks, C. Voacanga Extraction Manual Phase 4: Production and Purification of Ibogaine, 2015; pp1-26.

(16) Dogru, E.; Warzecha, H.; Seibel, F.; Haebel, S.; Lottspeich, F.; Stöckigt, J. The Gene Encoding Polyneuridine Aldehyde Esterase of Monoterpenoid Indole Alkaloid Biosynthesis in Plants Is an Ortholog of the $\alpha / \beta$ Hydrolase Super Family. Eur. J. Biochem. 2000, 267 (5), 1397-1406.

(17) Seong, S.; Lim, H.; Han, S. Biosynthetically Inspired Transformation of Iboga to Monomeric Post-Iboga Alkaloids. Chem. 2019, 5 (2), 353-363. 